\title{
Renal handling of prednisolone/prednisone: effect of steroid dose and 11ß-hydroxysteroid dehydrogenase
}

\author{
M. Conti, ${ }^{1}$ F. J. Frey, ${ }^{2}$ G. Escher, ${ }^{2}$ C. Marone ${ }^{1}$ and B. M. Frey ${ }^{2}$ \\ ${ }^{2}$ Division of Nephrology, University Hospital of Berne; and ${ }^{1}$ Department of Medicine, Ospedale S. Giovanni, \\ Bellinzona, Switzerland
}

\begin{abstract}
The purposes of this study were: (1) to determine under steady-state conditions whether the renal clearance of prednisolone is concentration dependent, and (2) to establish whether the urinary excretion of prednisolone and its biologically inactive 11-dehydro metabolite prednisone depend upon the activity of $11 \beta$-hydroxysteroid dehydrogenase (11ßOHSD). For that purpose 10 healthy volunteers were infused to steady state over a 13 -h period either at a low $(11 \mu \mathrm{g} / \mathrm{h} \times \mathrm{kg})$ or a high $(70 \mu \mathrm{g} / \mathrm{h} \times \mathrm{kg})$ rate with prednisolone on two occasions, once without and once with administration of glycyrrhetinic acid, an inhibitor of $11 \beta-O H S D$. Prednisolone and prednisone were measured by high-pressure liquid chromatography. Mean renal clearance values of total or unbound prednisolone were several times higher during the high than the low infusion rate. The fractional renal clearance of unbound prednisolone during the high, but not during the low infusion rate exceeded 1 . This indicates that in addition to unbound prednisolone, protein-bound prednisolone is excreted in urine at high plasma concentrations. Inhibition of $11 \beta-O H S D$ increased the urinary ratios of prednisolone/prednisone in all subjects. Conclusions: (1) The renal clearance of prednisolone is concentration dependent; (2) there must be tubular secretion and/or glomerular filtration of prednisolone bound to plasma proteins; (3) the urinary excretion of prednisolone/prednisone is modulated by the activity of $11 \beta-O H S D$.
\end{abstract}

Key words: clearance; $11 \beta$-hydroxy-steroid dehydrogenase; glycyrrhetinic acid; humans; prednisolone; prednisone

\section{Introduction}

For virtually all endo- or xenobiotics the total body clearance value is concentration independent or

Correspondence and offprint requests to: Brigitte $\mathrm{M}$. Frey, $\mathrm{PhD}$, Division of Nephrology, Freiburgstrasse 3, Inselspital, 3010 Berne, Switzerland. declines with increasing free concentrations as a consequence of saturation of the elimination process [1]. This is also the case for agents excreted mainly by the kidney, such as inulin, creatinine, para-aminohippurate or others. Prednisolone, however exhibits a peculiar kinetic feature: the total body clearance increases with increasing plasma concentrations of the steroid (for review see refs $[2,3])$. Part, but not all of the changes in clearance are related to changes in the plasma protein binding of prednisolone [4]. In addition increased excretion and/or metabolism of prednisolone with increasing concentrations must also be postulated as the second mechanism of the dose dependency. The assessment of the latter is complicated by the interconversion process between prednisolone and prednisone $[2,3]$. The organ(s) which might have the functional capability to enhance the clearance with increasing concentrations have not been identified. Therefore it is of general interest to establish whether the kidney could account for such an unusual concentrationdependent clearance. One purpose of the present investigation was thus to establish whether the renal clearance of prednisolone increases with increasing free prednisolone concentrations in plasma.

Cortisol is converted to its 11-dehydro metabolite, cortisone by the microsomal enzyme 11ß-OHSD [5]. Recently differential tissue distribution of $11 \beta-O H S D$ has been observed [6]. The tissue-specific distribution of $11 \beta$-OHSD is thought to confer specificity to mineralocorticoids in the presence of glucocorticoids in mineralocorticoid target tissues [7]. The activity of $11 \beta$-OHSD can be modulated by endo- and xenobiotics $[7,8]$. Among the xenobiotics liquorice extracts, derived from roots of the plant Glycyrrhizia glabra, used for decades as flavouring agents for food and beverages are probably the most relevant. It was demonstrated that glycyrrhetinic acid (GA), the hydrolytic product of glycyrrhizic acid exhibits mineralocorticoid activity by inhibiting the oxidation of the hydroxyl group at 11 th position of cortisol [9]. Cortisol is structurally related to prednisolone. It is assumed, that $11 \beta-O H S D$ accounts also for the conversion of the immunosuppressive agent prednisolone to the biologically 
inactive metabolite prednisone $[2,3]$. Therefore in the present investigation GA was administered during infusions of prednisolone in order to assess the impact of the inhibition of $11 \beta$-OHSD on the renal handling of prednisolone/prednisone at steady state.

\section{Subjects and Methods}

\section{Subjects}

Ten male volunteers, ranging in age from 20 to 30 years and weight from 55 to $75 \mathrm{~kg}$, participated in the study. The volunteers were not on chronic drug therapy. Two weeks before the study was initiated no food intake containing glycyrrhetinic acid derivatives were allowed. The subjects gave informed consent according to a protocol approved by the Ethics Committee at our institution.

\section{Protocol}

The volunteers were investigated on two occasions separated by 7 days. All studies were started between 07.00 and 08.00 . Catheters were placed in veins of each arm to allow simultaneous glucocorticoid, glucose, $\mathrm{NaCl}$, and $\mathrm{KCl}$ infusion and blood sampling. Prednisolone (Hydeltrasol ${ }^{(1)}$ ) was infused over a 13-h period at a rate of $70 \mu \mathrm{g} / \mathrm{kg} / \mathrm{h}$ following an i.v. bolus dose of $450 \mu \mathrm{g} / \mathrm{kg}$ in five subjects and at a rate of $11 \mu \mathrm{g} / \mathrm{kg} / \mathrm{h}$ following an i.v. bolus dose of $70 \mu \mathrm{g} / \mathrm{kg}$ in five additional subjects. In a previous investigation we demonstrated that by administering these doses a steady state of prednisolone and prednisone is achieved within $3 \mathrm{~h} \mathrm{[4].} \mathrm{The}$ volume of solute infused continuously over the 13-h study period was 21 containing a total of $66 \mathrm{~g}$ of glucose, $122 \mathrm{mmol}$ of sodium, $40 \mathrm{mmol}$ of potassium, and $162 \mathrm{mmol}$ of chloride. No food and fluid intake was allowed during that period. Six and $9 \mathrm{~h}$ after the beginning of the infusion two capsules containing each $200 \mathrm{mg}$ of GA or saccharose (placebo) were given. On each study day $5 \mathrm{ml}$ blood samples were collected just before steroid administration and 15, 60, 120, 180, 270, $315,360,420,510,600,690$, and 780 min after i.v. prednisolone dosing. Three urine collections were obtained on each study day (180-360 $\mathrm{min}, 420-600 \mathrm{~min}, 600-780 \mathrm{~min}$ ).

\section{Analytical methods}

Plasma samples were analysed for prednisolone and prednisone by a previously described HPLC procedure [10]. The intraday assay variability coefficient of variation (CV) of this method is $1-4 \%$, the interday assay CV $2.8-10.6 \%$ [10]. Equilibrium dialysis was performed to quantify the concentration of unbound prednisolone in plasma as described [11]. Because of the concentration-dependent protein binding of prednisolone, protein binding was determined in each plasma sample from each subject. The intraday variability for the protein binding measurements was found to be $1.2 \%$, the interday variability $1.6 \%$. The in-vitro fraction of unbound prednisolone was calculated by dividing the concentration in the buffer half-cell by the concentration in the plasma halfcell. Because prednisolone protein binding is non-linear it is necessary to correct the binding parameters for the changes in concentration that are caused by diffusion from plasma into buffer during equilibrium dialysis. This was done by the method of Behm and Wagner [12]. Prednisolone, prednisone and $6 \beta$-hydroxyprednisolone were assessed in urine using a previously described HPLC procedure [13] exhibiting an intraday $\mathrm{CV}$ of $5.5-10.9 \%$ and an interday $\mathrm{CV}$ of $4.5-11.6 \%[13]$.

\section{Calculations}

Renal clearance values of total and unbound prednisolone and prednisone were calculated by dividing the amount of prednisolone recovered in the urine by the corresponding plasma concentrations of the steroid. An estimate of the fractional renal clearance of the steroids was derived by dividing the renal clearance of the steroid by the glomerular filtration rate as assessed by the creatinine clearance. Nonrenal clearance (metabolic clearance) values were obtained by subtracting renal clearance from total body clearance values. Statistical analysis was performed with the help of the Statistical Analysis System software package (version OS 6.03, SAS Institute Inc., Carry, NJ). Comparison for variations over time was performed by analysis of variance, using the Hotelling-Lawley-Spur test, selecting the variation over time with GA versus placebo. All the values are given as mean $\pm S E$.

\section{Results}

Six hours after the bolus injection followed by the perfusion of prednisolone, prednisolone and prednisone concentrations in plasma were at steady state (Table 1). These concentrations remained constant up to $780 \mathrm{~min}$ when placebo was administered. The first dose of $400 \mathrm{mg} \mathrm{GA}$ was given at $360 \mathrm{~min}$, and the second dose at $540 \mathrm{~min}$ (Table 1, Figure 1). From 510 min until the end of the experiment, the prednisone concentrations in plasma steadily declined when GA, but not placebo, was given (Table 1). During the same time period, the concentrations of prednisolone increased when low doses, but not when high doses, of prednisolone were infused (Table 1). As a consequence, following the administration of GA, the prednisolone/prednisone concentration ratios in plasma increased (Fig. 1, open symbols).

The first urine collection was performed before (180-360 $\mathrm{min})$, and two additional collections after the administration of GA (420-600 min, 600-780 min) (Table 2). Following the low infusion of prednisolone, the rate of prednisolone excretion increased and that of prednisone decreased after the administration of GA. As a consequence, the urinary prednisolone/ prednisone concentration ratios increased after GA (Figure 1, dark columns). Furthermore, a decline in the urinary excretion rate of prednisone was seen after the high infusion rate of prednisolone (Table 2). Therefore, also the urinary prednisolone/prednisone ratios increased after the high infusion of prednisolone when GA was given (Figure 1, dark columns, $600-780 \mathrm{~min}$ ). The increase of the urinary ratio of prednisolone/prednisone was present in all subjects $(P<0.05)$ and more pronounced after the low than after the high dose of prednisolone (Figure 1).

At each time-point the mean prednisolone/prednisone concentration ratio (without GA) in plasma was higher during the high than during the low infusion 


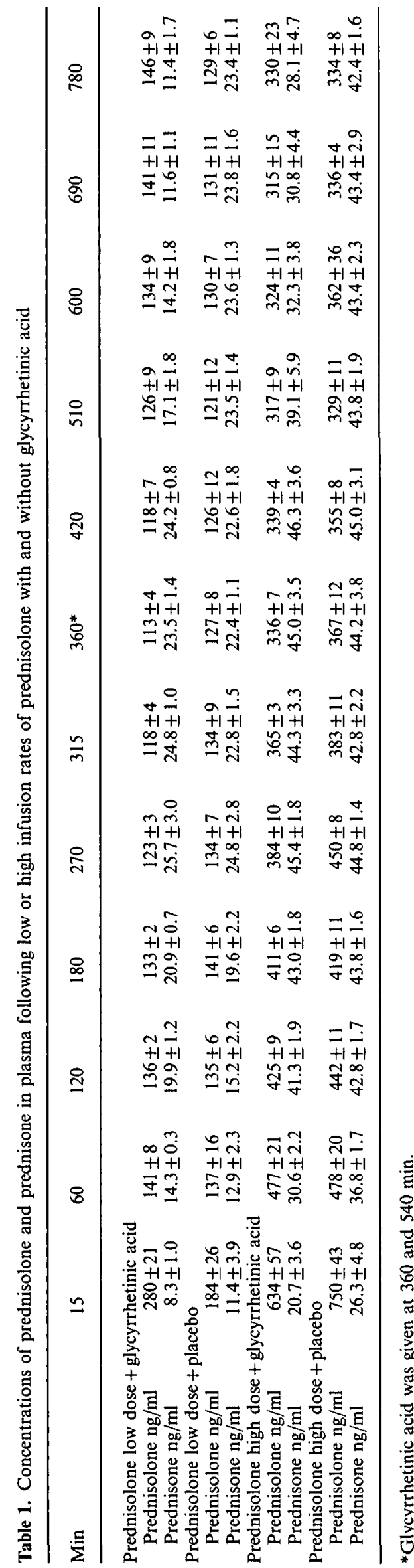

rate of prednisolone $(P<0.001)$ (Figure 1, open symbols). A similar dose dependency of the prednisolone/ prednisone ratio was found for urine (Figure 1, open columns). The mean values of these ratios in urine were more than three times higher during the high than during the low infusion rate of prednisolone (Figure 1, open columns). The maximum effect of GA on the prednisolone/prednisone concentration ratios was observed only after several hours, an observation in line with the time to peak concentration of GA following dosing of the inhibitor [14].

GA did not change the total body clearance of total or unbound prednisolone (low dose, without versus with GA: $1.4 \pm 0.1$ versus $1.3 \pm 0.1 \mathrm{ml} / \mathrm{min} \times \mathrm{kg}$; high dose, without versus with $\mathrm{GA}: 3.4 \pm 0.1$ versus $3.6 \pm 0.2 \mathrm{ml} / \mathrm{min} \times \mathrm{kg}$ ). Similarly, GA did not influence the total body clearance of prednisolone when calculated with reference to unbound steroid concentrations (low dose, without versus with GA: $8.8 \pm 0.9$ versus $8.2 \pm 0.5 \mathrm{ml} / \mathrm{min} \times \mathrm{kg}$; high dose, without versus with GA: $15.0 \pm 0.9$ versus $15.7 \pm 1.8 \mathrm{ml} / \mathrm{min} \times \mathrm{kg}$ ). In all subjects given the high infusion rate of prednisolone, the clearance values were higher than those during the low infusion rate. All the aforementioned clearance values were calculated by considering the time period between 600 and $780 \mathrm{~min}$. Similar results were obtained when the earlier time periods $(180-360 \mathrm{~min}$ or 420-600 min, Figure 1) were analysed.

The renal clearance of prednisolone and prednisone increased with increasing steroid dose (Figure 2, Table 3). This concentration dependency was not due to an altered glomerular filtration rate because the corresponding fractional renal clearance values followed the same dose dependency. The values of the fractional renal clearance of prednisolone following the high dose of prednisolone were higher than one in all subjects (Table 3). The renal clearance values of prednisolone obtained from the low-dose study increased after the administration of GA (Figure 2).

\section{Discussion}

Prednisolone exhibits concentration-dependent kinetics, i.e. an increase in concentration results in an increase in plasma clearance of prednisolone, as shown in the present investigation [3,4]. Legler et al. presented evidence that the changes in clearance are mostly related to changes in the plasma protein binding of prednisolone [4]. Prednisolone binds to transcortin and albumin in a non-linear manner, with the percentage of unbound drug increasing with increasing steroid concentration [3]. The relevance of protein binding for the dose-dependency of prednisolone kinetics is supported by the observation that subjects exhibiting an abnormally high binding of prednisolone to plasma proteins (women taking oestrogen-containing oral contraceptive steroids) have decreased values of total body clearance when calculated with reference to total prednisolone concentrations, whereas individuals exhibiting an abnormally low binding of prednisolone 
Low Dose

Prednisolone / Prednisone Concentration Ratios
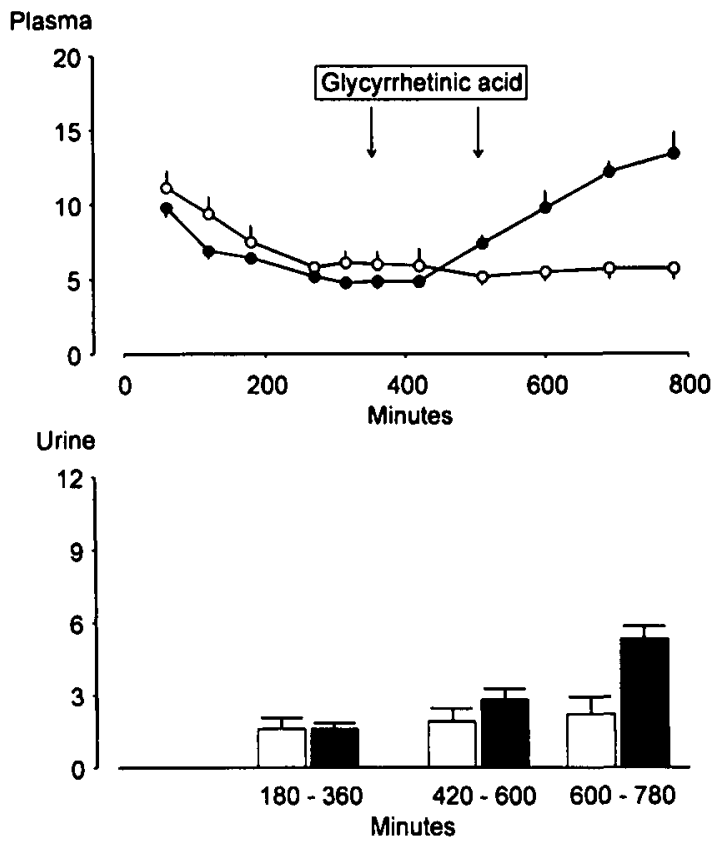

$0-G A$

$\bullet+G A$
High Dose
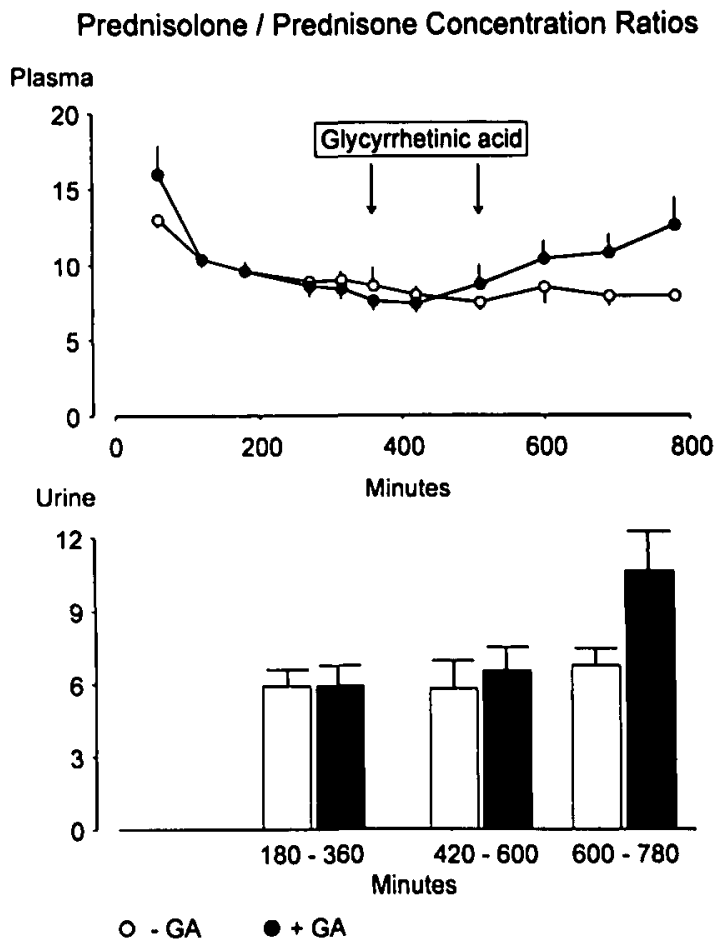

Fig. 1. Prednisolone/prednisone concentration ratios in plasma and urine following a low (left) and a high (right) infusion rate of prednisolone. The arrows indicate the intake of glycyrrhetinic acid or placebo. The open circles and columns represent results obtained following placebo and the closed circles and columns those following glycyrrhetinic acid. Prednisolone/prednisone ratios were higher following the high than the low infusion rate, both in plasma and in urine. The plasma ratios increased in all subjects $(P<0.05)$ after 510 min and $600 \mathrm{~min}$ following the low and the high infusion rate respectively. The urinary ratios increased after glycyrrhetinic acid in all subjects investigated.

Table 2. Prednisolone and prednisone in urine after low or high infusion rates of prednisolone with or without glycyrrhetinic acid

\begin{tabular}{|c|c|c|c|}
\hline Min & $180-360^{*}$ & $420-600^{* *}$ & $600-780^{* *}$ \\
\hline \multicolumn{4}{|c|}{ Prednisolone low dose + glycyrrhetinic acid } \\
\hline Prednisolone $\mu \mathrm{g} / 3 \mathrm{~h}$ & $195 \pm 29$ & $286 \pm 42$ & $376 \pm 46$ \\
\hline Prednisone $\mu \mathrm{g} / 3 \mathrm{~h}$ & $131 \pm 25$ & $106 \pm 16$ & $71 \pm 9$ \\
\hline \multicolumn{4}{|c|}{ Prednisolone low dose + placebo } \\
\hline Prednisolone $\mu \mathrm{g} / 3 \mathrm{~h}$ & $248 \pm 53$ & $190 \pm 50$ & $158 \pm 32$ \\
\hline Prednisone $\mu \mathrm{g} / 3 \mathrm{~h}$ & $165 \pm 11$ & $111 \pm 22$ & $100 \pm 15$ \\
\hline \multicolumn{4}{|c|}{ Prednisolone high dose + glycyrrhetinic acid } \\
\hline Prednisolone $\mu \mathrm{g} / 3 \mathrm{~h}$ & $3241 \pm 312$ & $2503 \pm 89$ & $2953 \pm 340$ \\
\hline Prednisone $\mu \mathrm{g} / 3 \mathrm{~h}$ & $545 \pm 33$ & $405 \pm 49$ & $306 \pm 69$ \\
\hline \multicolumn{4}{|c|}{ Prednisolone high dose + placebo } \\
\hline Prednisolone $\mu \mathrm{g} / 3 \mathrm{~h}$ & $2619 \pm 392$ & $2561 \pm 346$ & $2737 \pm 310$ \\
\hline Prednisone $\mu \mathrm{g} / 3 \mathrm{~h}$ & $434 \pm 30$ & $458 \pm 59$ & $389 \pm 31$ \\
\hline
\end{tabular}

* Before glycyrrhetinic acid; ** after glycyrrhetinic acid.

to plasma proteins (nephrotic patients) have increased values of these parameters [15-18].

In addition to the concentration-dependent plasma protein binding, the concentration-dependent total body clearance of unbound prednisolone also accounts for part of the concentration-dependent clearance of total prednisolone as shown by Legler et al. [4]. The present study reveals that part of the concentrationdependent total body clearance of unbound steroids is due to a rise in renal clearance with increasing concentrations of prednisolone (Figure 2). The dosedependent renal clearance cannot be explained by a dose-dependent impact of the corticosteroid on the glomerular filtration rate, because the ratios of renal clearance of total or unbound prednisolone to the creatinine clearance (fractional renal clearance) were higher after the high than after the low dose of the steroid.

The observed renal clearance values of prednisolone are not consistent with the involvement of glomerular filtration of unbound prednisolone alone. In addition to glomerular filtration of unbound prednisolone there must be filtration and/or active tubular secretion of prednisolone bound to plasma proteins. Compared to transcortin, albumin exhibits $10^{4}$ times lower affinity for prednisolone binding [18]. Thus prednisolone bound to albumin rather than prednisolone bound to transcortin may be filtered or secreted. At low concentrations most prednisolone is bound to transcortin. With increasing concentrations a larger fraction is bound to albumin, thus increasing the availability for excretion of prednisolone with increasing plasma concentrations.

An alternative explanation for the increase of the prednisolone clearance with increasing concentrations of prednisolone might be a saturation of the $11 \beta$ OHSD at high prednisolone concentrations. In line with that hypothesis is the observation that during the 
Renal Clearance of Prednisolone

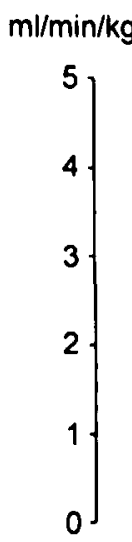

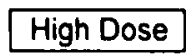

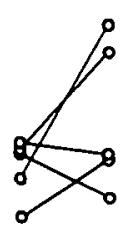

Fractional Renal Clearance of Prednisolone

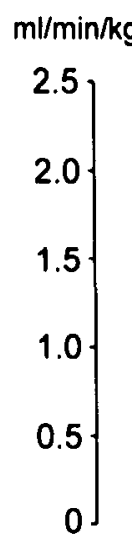

\section{High Dose}
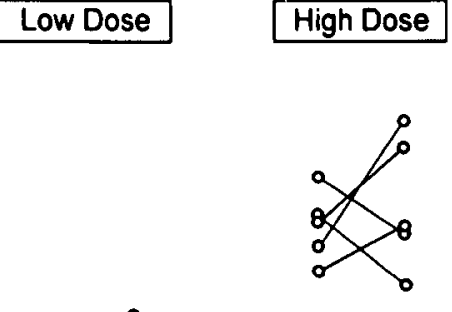

Fig. 2. Renal clearance and fractional renal clearance of unbound prednisolone before and after glycyrrhetinic acid (GA) during low or high infusion rates of prednisolone. Both clearance values were higher in all subjects when calculated during the high than during the low infusion rate of prednisolone. Glycyrrhetinic acid increased these values in eight, of 10 subjects.

Table 3. Renal clearance of prednisolone and prednisone following high and low infusion rates of prednisolone without and with glycyrrhetinic acid

\begin{tabular}{|c|c|c|c|c|c|c|}
\hline \multirow[t]{2}{*}{ Measurements } & \multicolumn{3}{|c|}{ Without glycyrrhetinic acid } & \multicolumn{3}{|c|}{ With glycyrrhetinic acid } \\
\hline & High dose & Low dose & $P$ values & High dose & Low dose & $P$ values \\
\hline \multicolumn{7}{|c|}{ Renal clearance $(\mathrm{ml} / \mathrm{min} \times \mathrm{kg})$ : } \\
\hline Total prednisolone & $0.65 \pm 0.03$ & $0.10 \pm 0.02^{\mathrm{a}}$ & $<0.05$ & $0.82+0.10$ & $0.21 \pm 0.02^{\mathrm{a}}$ & $<0.05$ \\
\hline Unbound prednisolone & $2.87 \pm 0.15$ & $0.58 \pm 0.05^{\mathrm{b}}$ & $<0.05$ & $3.45 \pm 0.38$ & $1.30 \pm 0.12^{\mathrm{b}}$ & $<0.05$ \\
\hline Prednisone & $0.80 \pm 0.06$ & $0.33 \pm 0.05$ & $<0.05$ & $0.96 \pm 0.26$ & $0.49 \pm 0.04$ & n.s. \\
\hline \multicolumn{7}{|l|}{ Creatinine clearance } \\
\hline \multirow{2}{*}{\multicolumn{7}{|c|}{ Fractional renal clearance: }} \\
\hline & & & & & & \\
\hline Total prednisolone & $0.37 \pm 0.02$ & $0.06 \pm 0.01^{\mathrm{c}}$ & $<0.05$ & $0.42 \pm 0.05$ & $0.14 \pm 0.02^{\mathrm{c}}$ & $<0.05$ \\
\hline Unbound prednisolone & $1.61 \pm 0.09$ & $0.35 \pm 0.04^{\mathrm{d}}$ & $<0.05$ & $1.75 \pm 0.17$ & $0.76 \pm 0.04^{\mathrm{d}}$ & $<0.05$ \\
\hline Prednisone & $0.45 \pm 0.04$ & $0.20 \pm 0.04$ & $<0.05$ & $0.49 \pm 0.13$ & $0.30 \pm 0.03$ & n.s. \\
\hline
\end{tabular}

Plus-minus values are means $\pm \mathrm{SE} ; \mathrm{a}, P<0.02 ; \mathrm{b}, P<0.01 ; \mathrm{c}, P<0.02 ; \mathrm{d}, P<0.001$.

low infusion rate of prednisolone, the renal clearance increased in all subjects after the administration of GA (Figure 2). A similar increase was observed in three of five subjects during the high infusion with prednisolone (Figure 2). The explanation of the saturation of $11 \beta$ OHSD at high concentrations of prednisolone, however, cannot explain completely the dose dependent clearance of prednisolone for two reasons. First, the inhibition of $11 \beta$-OHSD by GA did not increase the renal clearance of prednisolone up to the level of high dose without GA (Figure 2). Second, if the inhibition of the conversion of prednisolone to prednisone was relevant for the prednsiolone clearance, one might predict a decline of the renal clearance of prednisone after the administration of GA, which was not the case (Table 3).

In the past, kinetics of prednisolone have usually been assessed by administering a single oral dose of prednisone or an i.v. bolus dose of prednisolone; thus, the concentration measurements were not made at steady state. In some of these studies the urinary excretion of prednisolone was measured. These measurements allowed a rough estimate of the renal handling of prednisolone. Interestingly, the fractional renal clearance of unbound prednisolone after an i.v. bolus dose of $0.8 \mathrm{mg} / \mathrm{kg}$ of prednisolone was reduced in patients with nephrotic syndrome [17], subjects older than 65 years [19] or in renal transplant patients [20,21] when compared with normal healthy volunteers. The mean values of the fractional renal clearance of unbound prednisolone were about 2.25 in healthy volunteers, 1.25 in nephrotic patients, 0.98 in elderly subjects, 0.75 in renal transplant patients 1 year after transplantation with a steady decline to a value of 0.5 over the next 3-5 years following successful kidney transplantation.

In the two groups of patients and in the elderly subjects the creatinine clearance was slightly reduced 
(mean value around $65 \mathrm{ml} / \mathrm{min}$ ). This indicates that patients with impaired renal function for various reasons excrete relatively less prednisolone in urine. It is conceivable that the transport system for prednisolone is impaired and/or the conversion of prednisolone into prednisone by $11 \beta$-OHSD enhanced. The slightly decreased urinary concentration ratios of prednisolone/ prednisone, observed in elderly subjects, nephrotics, and renal transplant patients, are compatible with the latter view. Evidence for a decrease of $11 \beta-O H S D$ activity in patients with impaired renal function has previously already been shown by Whitworth et al. by determining cortisol/cortisone ratios in plasma from patients with renal diseases [22].

The observation of increased urinary prednisolone, despite there being no change in plasma levels, has previously been found for cortisol following GA [23]. The apparent contradiction of an unchanged total body clearance in the presence of an increased renal clearance following GA is best explained by the observation that GA inhibits other enzymes involved in the catabolism of corticosteroids [24]. Nevertheless the absence of an effect of GA on the total body clearance of prednisolone is partly at variance with a study of Chen et al. [25]. These investigators found also no impact of glycyrrhizin on the total prednisolone concentrations in plasma; however, they reported slightly higher concentrations of unbound prednisolone. Chen et al. measured only significantly higher unbound prednisolone concentrations with than without glycyrrhizin between the 4 th and the 8 th hour after prednisolone dosing, but not during the initial $4 \mathrm{~h}$. Thus it is conceivable that at very low prednisolone concentrations glycyrrhizin reduces the total body clearance of prednisolone. Alternatively the difference between the results of Chen et al. and those from the present investigation may be explained by differences in study design. Chen et al. administered a very low dose of prednisolone (about $5 \mathrm{mg}$ ), injected glycyrrhizin intravenously, and used a method for measuring the free fraction of prednisolone that did not consider the non-linear binding of prednisolone to plasma proteins. Whether glycyrrhizin affected the interconversion of prednisolone to prednisone or the renal clearance of prednisolone is unknown, because the method did not allow assessment specifically of prednisone and prednisolone, and no urinary measurements were performed [25].

GA modulates the activity of $11 \beta-O H S D$. Inhibition of that enzyme might account for local changes of the concentration ratio between biological active prednisolone and inactive prednisone and by that mechanism modulate mineralocorticoid and glucocorticoid effects. We have recently shown that the prednisolone/prednisone concentration ratio was about 40 times higher in liver than in kidney tissue and 5- to 15 -fold higher in lung, heart, spleen, or plasma than in kidney from rats [26]. Inhibition of $11 \beta$-OHSD by GA increased the prednisolone/prednisone ratio in kidney and other tissues [26]. This indicates that the increase of prednisolone/prednisone in urine and plasma following GA observed in humans in the present study reflect corresponding changes in tissues.

Acknowledgements. Supported by the Swiss National Foundation for Scientific Research (Grant Nr. 32-31048-91), the Stanley Thomas Johnson Foundation, and the Stiftung zur Förderung der Forschung an der Hochschule Bern. We acknowledge the statistical expertise of Dr P. Ferrari.

\section{References}

1. Gerber N, Wagner JG. Explanation of dose-dependent decline of diphenylhydantoin plasma levels by fitting to the integrated form of Michaelis-Menten equation. Res Comm Chem Pathol Pharmacol 1972;3:455-460

2. Frey FJ. Nephrology forum: pharmacokinetic determinants of cyclosporine and prednisone in renal transplant patients. Kidney Int 1991;39:1034-1050

3. Frey FJ. Kinetics and dynamics of prednisolone. Endocrinol Rev 1987;8:453-473

4. Legler UF, Frey FJ, Benet LZ. Prednisolone clearance at steady state in man. $J$ Clin Endocrinol Metab 1982;55:762-767

5. Lakshmi V, Monder C. Evidence for independent 11-oxidase and 11-reductase activities of $11 \beta$-hydroxysteroid dehydrogenase; enzyme latency, phase transitions, and lipid requirements. Endocrinology 1985;116:552-560

6. Bonvalet JP, Doignon I, Blot-Chabaud M, Pradelles P, Farman $N$. Distribution of $11 \beta$-hydroxysteroid dehydrogenase along the rabbit nephron. J Clin Invest 1990;86:832-837

7. Funder JW, Pearce PT, Smith R, Smith AI. Mineralocorticoid action: target tissue specificity is enzyme, not receptor, mediated. Science 1988;242:583-585

8. Bühler $\mathrm{H}$, Perschel $\mathrm{FH}$, Hierholzer $\mathrm{K}$. Inhibition of rat renal $11 \beta$-hydroxysteroid dehydrogenase by steroidal compounds and triterpenoids; structure/function relationship. Biochim Biophys Acta 1991;1075:206-212

9. Edwards CRW, Burt D, Mcintyre MA et al. Localisation of 11 $\beta$-hydroxysteroid dehydrogenase-tissue specific protection of the mineralocorticoid receptor. Lancet 1988:29:986-989

10. Frey FJ, Frey BM, Benet LZ. Liquid chromatographic measurement of endogenous and exogenous glucocorticoids in plasma. Clin Chem 1979;25:1944-1947

11. Frey FJ, Frey BM, Greither A, Benet LZ. Prednisolone clearance at steady-state in dogs. J Pharmacol Exp Ther 1980;215:287-291

12. Behm JHL, Wagner JG. Errors in interpretation of data from equilibrium dialysis protein binding experiments. Res Commun Chem Pathol Pharmacol 1979;26:145-160

13. Frey BM, Frey FJ. Simultaneous measurement of prednisone prednisolone and 6 $\beta$-hydroxyprednisolone in urine by high performance liquid chromatography coupled with a radioactivity detector. J Chromatogr 1982;229:283-292

14. Krähenbühl S, Hasler F, Frey BM, Frey FJ, Brenneisen R, Krapf R. Kinetics and dynamics of orally administrated $18 \beta$ glycyrrhetinic acid in humans. $J$ Clin Endocrinol Metab 1994;78:581-585

15. Boekenoogen SJ, Szefler SJ, Jusko WJ. Prednisolone disposition and protein binding in oral contraceptive users. J Clin Endocrinol Metab 1983;56:702-709

16. Bergrem H. Pharmacokinetics and protein binding of prednisolone in patients with nephrotic syndrome and patients undergoing hemodialysis. Kidney $I n t$ 1983;23:876-881

17. Frey FJ, Frey BM. Altered plasma protein-binding of prednisolone in patients with the nephrotic syndrome. Am J Kidney Dis 1984;3:339-342

18. Frey BM, Frey FJ. The effect of altered prednisolone kinetics in patients with the nephrotic syndrome and in women taking oral contraceptive steroids on human mixed lymphocyte cultures. $J$ Clin Endocrinol Metab 1985;60:361-369

19. Stuck AE, Frey BM, Frey FJ. Kinetics of prednisolone and endogenous cortisol suppression in the elderly. Clin Pharmacol Ther 1988;43:354-361

20. Frey FJ, Schaad HJ, Renner EL, Horber FF, Frey BM, Preisig 
R. Liver function in stable renal transplant patients. Hepatology 1989;9:606-613

21. Schaad HJ, Frey BM, Renner EL, Preisig R, Frey FJ. Microsomal liver function declines steadily after kidney grafting: a 3-5 yr follow up. Kidney Int 1992;41:420-427

22. Whitworth JA, Stewart PM, Burt D, Atherden SM, Edwards CRW. The kidney is the major site of cortisone production in man. Clin Endocrinol 1989;31:355-361

23. Stewart PM, Valentino R, Wallance AM, Burt D, Shackelton CHL, Edwards CRW. Mineralocorticoid activity of liquorice: $11 \beta$-hydroxysteroid dehydrogenase deficiency comes of age. Lancet 1987;2:821-823
24. Latif SA, Conca TJ, Morris DJ. The effects of the licorice derivative, glycyrrhetinic acid, on hepatic 3alpha- and $3 \beta$-hydroxysteroid dehydrogenases and 5alpha and $5 \beta$-reductase pathways of metabolism of aldosterone in male rats. Steroids 1990;55:52-58

25. Chen MF, Shimada F, Kato H, Yano S, Kanaoka M. Effect of glycyrrhizin on the pharmacokinetics of prednisolone following low dosage of prednisolone hemisuccinate. Endocrinol Jpn 1990;37:331-341

26. Escher G, Frey FJ, Frey BM. 11 1 -hydroxysteroid dehydrogenase accounts for low prednisolone/prednisone ratios in the kidney. Endocrinology, 1994;135:101-106.

Received for publication 27.1.94

Accepted in revised form 27.6 .94 